\title{
Cuckoo Search and M-Tree based Multicast Ad hoc On-demand Distance Vector Protocol for MANET
}

\author{
D. Madhu Babu, M. Ussenaiah
}

\begin{abstract}
The proposed work, Cuckoo Search (CS) and M-Tree based Multicast Ad hoc On-demand Distance Vector (MAODV), is a two-step process, which involves M-Tree construction and optimal multicast route selection. Divisional based Cluster (DIVC), a technique of clustering inspired from Divisive clustering, builts the M-Tree using three constraints, destination flag, path-inclusion factor, and multi-factor. This paper aims to provide optimal multicasting with multiple objectives, such as energy, link lifetime, distance and delay.

Index Terms - Cuckoo Search, Divisive clustering, MANET, M-Tree, , Multicast Routing,
\end{abstract}

\section{INTRODUCTION}

Mobile Ad hoc Networks (MANETs) [21] are infrastructure-less and self-organized wireless networks that have a decentralized management. The multi-hop wireless links in the MANET let the randomly moving nodes to exchange the data among themselves. Communication between two nodes can be built with the intermediate nodes, even if the nodes are away from the radio range [6, 24]. Although the nodes can construct a network with its selforganizing ability, these mobile nodes have restrictions in the utilization of resources, transmission power, and battery life [21].

In MANET routing, the two main classifications include proactive and reactive routing protocols. The proposed work is for reactive multicast routing protocol whose unique feature lead to the discovery of different protocols and algorithms for routing $[12,13]$. In this kind of routing, the source node transmits the data packets to a set of destination nodes, which is a common issue in the communication networks. The multicast routing protocols aims to achieve on time data delivery, reduced routing control overheads and stable connectivity with reduced packet losses $[9,15]$.

This paper proposes a multicast routing protocol, CSMAODV for MANET. This tree-based clustering and optimization algorithm for routing is concerned with objectives namely, energy, LLT, distance, and delay. The proposed multicast routing approach consists of two stages:

i. Construction of M-Tree using DIVC

ii. Finding optimal multicast routes using CS algorithm.

The rest of the paper organised as follows:

Revised Manuscript Received on July 10, 2019.

D. Madhu Babu, Research Scholar, Department of Computer Science, Vikrama Simhapuri University, Nellore, AP, India. (E-mail: dmadhubabu2013@gmail.com)

Dr. M. Ussenaiah, Assistant Professor, Department of Computer Science , Vikrama Simhapuri University, Nellore, AP, India. (E-mail: ussenaiah.malligala @gmail.com)

$\begin{array}{lll}\text { Section-ii } & : & \text { DIVC-based m-tree formation } \\ \text { Section-iii } & : & \text { CS - based multicast routing } \\ \text { Section-iv } & : & \text { Cuckoo search algorithm } \\ \text { Section-v } & : & \text { Conclusion of the paper }\end{array}$

\section{DIVC-BASED M-TREE FORMATION}

DIVC is a clustering technique inspired by Divisive Clustering or top-down approach. Here, the technique groups all the data in a cluster, which then divides further into clusters until all the data forms a cluster separately. The division of clusters requires several criteria or constraints, which a node in the cluster satisfies. The algorithm considers three constraints, namely, multi-factor, path inclusion factor, and destination flag to form the clustering and thereby, to construct the M-Tree. When a source requires sending a data packet to the destination set, it transmits a RQ packet to its neighboring nodes in the transmission range. Each node checks whether the three constraints are satisfied to forward the data. Only the nodes that satisfy the criteria can take part in the communication. Consider a sample network topology for the clustering as in figure 1.

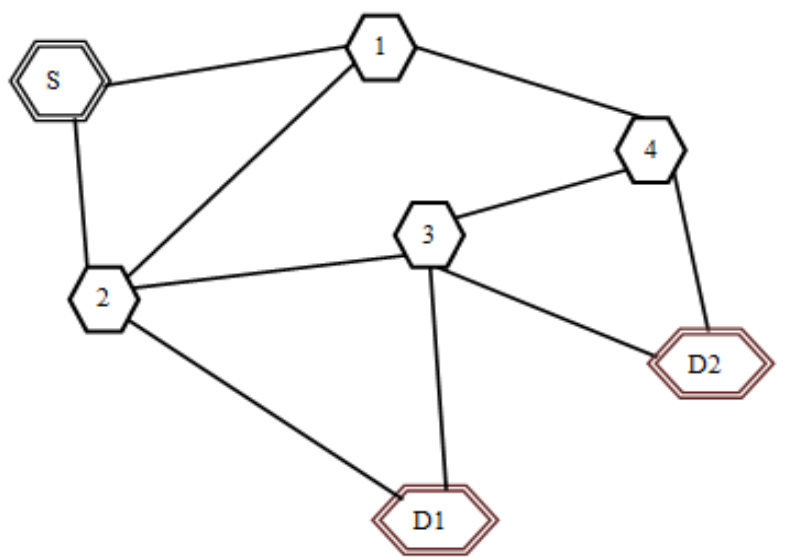

Figure 1. A sample network with two destinations

The following are the brief description of the constraints to build the M-Tree effectively.

Destination flag: The first constraint in DIVC is the destination flag, which notifies the destination node during the transmission to select the route. During the route

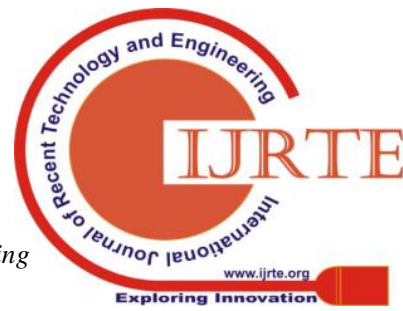




\section{CUCKOO SEARCH AND M-TREE BASED MULTICAST AD HOC ON-DEMAND DISTANCE VECTOR PROTOCOL FOR MANET}

discovery process, the technique selects the node by checking if it is a destination node using a flag. If the node is a destination node, the destination flag is set one and creates a path. In the other case, the flag is set zero and the node broadcasts RQ until it reaches the destination.

Path-inclusion factor: The path inclusion factor identifies the node in a path that has been involved in the transmission once. Thus, the factor can avoid the repeating paths, thereby reducing the processing time and wrong route formation.

With a preset value, this metric either considers or neglects the node/nodes along the path.

Multi-factor: The multi-factor of the DIVC approach consists of three different factors, such as energy of the node, LLT and the distance between two nodes in a cluster, given as,

$$
\text { Multi-factor }=\left[E_{\text {node }}+\frac{L L T}{N_{L L T}}+\left[1-\frac{D_{T}}{1-N_{D_{T}}}\right]\right] * \frac{1}{3}
$$

where, $E_{\text {node }}$ is the energy of a node, $L L T$ is the lifetime of a link between the nodes, $N_{L L T}$ is the normalized $L L T$, $D_{T}$ is the distance between two nodes and $N_{D_{T}}$ is the normalized distance. The formulation of multi-factor is such that the energy utilized is maximum, maximum $L L T$ and minimum distance. The functionality of $N_{L L T}$ and $N_{D_{T}}$ is to maintain a low value so that the lifetime of the network is maximized and the distance is reduced between the nodes.

Satisfying the above-explained constraints, a source node sends the RQ packet to the destination nodes via the intermediate nodes. Once the receiver receives the RQ packet, the nodes in the destination initiate the RP phase. The destination nodes begin to send the RP packet to the source node using the path reverse information in the RP packet. The intermediate nodes that receive the packet forward it to the neighboring packets until the source node receives the RP packet. Thus, the clustering technique constructs the M-Tree with the routes established between the source and the destinations. Figure 2 shows the M-Tree constructed using DIVC for the sample network.

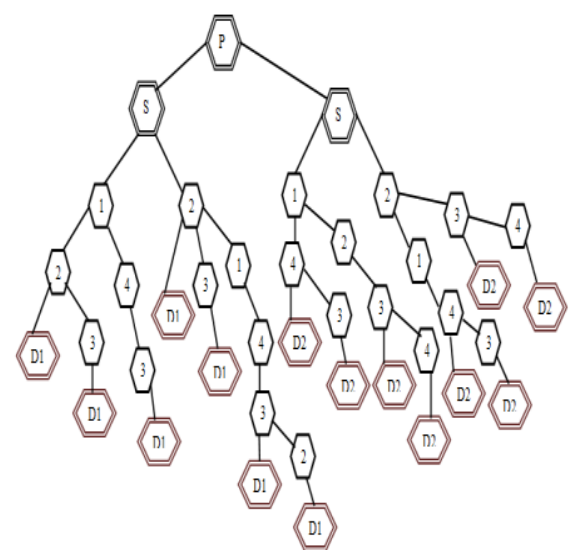

Figure 2. M-Tree Construction

\section{CS - BASED MULTICAST ROUTING}

The optimal selection of multicast routing to transmit the data packets is illustrated in this section. The multicasting selection scheme adopted here is based on CS algorithm to improve the search process. CS algorithm is an optimization technique that adopts the behavior of Cuckoo species with the Levy flight. The fitness evaluation of CS algorithm includes several objectives to form an effective route for the transmission. The following sections describe the solution encoding, fitness evaluation and the multicast routing algorithm in detail.

\subsection{Solution Encoding}

Solution encoding is the simplest way of representing the algorithmic procedure. The aim of a multicast routing algorithm is to broadcast the data packets from the source node to the desired number of destinations. Let $D_{1}$ and $D_{2}$ denote two destinations, which receive the information from the source $S$. Each destination has a number of paths to reach the destinations from the source, represented as, $D_{1}=P_{1}, P_{2}, \ldots, P_{m}$ and $D_{2}=P_{1}, P_{2}, \ldots, P_{n}$, such that the range of a path $P_{m}$ depends on the number of nodes in the corresponding path i.e. $O \leq P_{m} \leq N_{j}$. From the $m$ number of paths, the routing protocol points out the paths with better fitness values as the optimum solutions. Figure 3 displays the solution encoding of CS-based routing scheme.

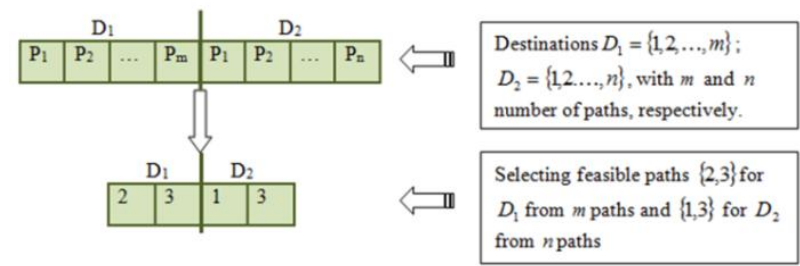

Figure 3. Solution Encoding of CS-based routing

\subsection{Fitness Formulation}

The fitness is an important function that determines the quality of the solution. The fitness of the algorithm considers four objectives energy, LLT, distance, and delay. The fitness of the algorithm must have maximum node energy, maximum LLT, reduced delay with minimum distance between the nodes, as in equation (15),

$$
F=\left(\frac{f_{1}}{2 T}\right)+\left\{\frac{1}{3 * f_{1} * 2}\left[\sum_{i=1}^{D} \sum_{j=1}^{P_{i}} \sum_{n=1}^{N_{j}}\left(E_{n}+\frac{L L T_{(n, n+1)}}{N_{L L T}}+\left[1-\frac{D_{T(n, n+1)}}{N_{D_{T}}}\right]\right)\right]\right\}
$$

where, $T$ is the total number of nodes in the network, $D$ is the number of destination, $P_{i}$ is the number of paths available to reach the $i^{\text {th }}$ destination, $E_{n}$ is the energy of the node, $L L T$ is the lifetime of the link that exists between the nodes $n$ and $n+1, D_{T}$ is the distance between $n$ and $n+1 . f_{1}$ is the total number of nodes available in a path, which is given as,

$$
f_{l}=\sum_{i=1}^{D} \sum_{j=1}^{P_{i}} \sum_{t=1}^{N_{j}}\left|N_{j}\right|
$$

\section{Published By:}


where, $N_{j}$ is the total number of nodes in the $j^{\text {th }}$ path.

An effective algorithm requires maximum node energy so that the node can participate in the transmission. The energy of the node often reduces due to the mobility and dynamic network topology. Hence, it is essential to maintain maximum energy in a node throughout the transmission. The nodal energy $E_{n}$ has a value in the range 0,1 , where 1 refers to the best case and 0 refers to the worst case.

The second constraint is the lifetime of the link (depends on the node's mobility), which an algorithm requires to be in maximum. LLT influences the lifetime of the route to improve the network lifetime. It also resembles the energy of the nodes in the link, which requires a maximum value to increase the LLT. Thus, the link that exists between two nodes $n$ and $n+1$ must attain a value, 0 or 1 , indicating the best and the worst value, respectively. The LLT calculation uses a normalized factor $N_{L L T}$ to normalize the LLT value in the range 0,1 .

The final objective is the distance metric $D_{T}$ between the nodes $n$ and $n+1$. Even though, the position of the nodes changes frequently, the distance between the nodes should be kept to a minimum. Similar to the other constraints, the distance measure also has a value that ranges from 0 to 1 . $N_{D_{T}}$ is the normalized distance that maintains $D_{T}$ in between 0 and 1. The delay of the algorithm is not formulated as it is directly proportional to the number of nodes connected in a route. Hence, the delay of the proposed algorithm increases with the increased number of nodes.

\section{CUCKOO SEARCH ALGORITHM \&} ANALYTICAL RESULTS

The proposed multicast routing algorithm is developed based on CS algorithm that depends on the habitat of cuckoo species. The algorithm considers three rules: i) At a time, the bird lays one egg, which represents a solution, on a randomly chosen host nest, ii) The high-quality egg or the solution with the best fitness proceeds the algorithm, iii) The host bird abandons the alien egg with low quality, while choosing the best with a probability. With the utilization of the newly formulated fitness function in the CS algorithm approach, optimal multicast routing of MANET is possible. Various steps of the algorithm are explained as follows,

\section{i. Initialization}

The algorithm generates an initial population $U_{i} ; O \leq U_{i} \leq K$, of dimension $K$. The objective is to select better solutions from the $K$ available paths, where the initial solution is,

$$
U_{i}=U_{i}^{1}, U_{i}^{2}, \ldots, U_{i}^{m}
$$

where, $m$ is the required number of paths.

\section{ii. Generation of solutions}

In the second stage, CS algorithm generates new solutions using the levy flight equation, which forms the appropriate solution initially as,

$$
X_{l}=X_{l-1}+Z \oplus \text { Levy } \lambda ; Z>0
$$

where, $Z$ denotes the step size, $\oplus$ is the entry-wise multiplication, $X_{l-l}$ is the solution at iteration $l-1$ and Levy $\lambda$ is the Levy flight model.

Levy flight randomly generates new solutions in each iteration, to select the most appropriate one. It follows a distribution given by,

$$
\text { Levy } \sim l^{-\lambda} ; \lambda=1,2,3
$$

where, $\lambda$ is the mean. For the solution updated based on equation (18), the newly computed objective function computes the fitness.

\section{iii. Multi-constrained Fitness Evaluation}

The fitness function formulated using the four objectives. The solution having maximum fitness is chosen as the best fit solution. The solution that has minimum node distance, maximum nodal energy and LLT with reduced delay is the best fit solution.

\section{iv. Termination}

Every algorithm requires a termination condition once it finds the best solution. The user can define the termination criteria according to the desired application of the algorithm. This optimization algorithm halts when the number of iterations reaches its maximum or when no significant change is observed in the results in further iterations.

Frame 1 exposes the pseudocode of the CS algorithm, which provides an outlook of the procedure.

\begin{tabular}{|l|l|}
\hline \multicolumn{2}{|l|}{ CS Algorithm } \\
\hline 1 & Input: Population $U_{i}$ \\
\hline 2 & Output: Best solution $X_{l}$ \\
\hline 3 & Begin \\
\hline 4 & Initialize the population $U_{i}$ of $K$ multiple solutions \\
\hline 5 & Generate a random solution using equation $(17)$ \\
\hline 6 & Update the solution using equation $(18)$ \\
\hline 7 & $\begin{array}{l}\text { Calculate the fitness } F\left(U_{i}\right) \text { for the random solution } \\
\text { and } F\left(X_{l}\right) \text { for the new solution using equation }(15)\end{array}$ \\
\hline 8 & if $F\left(U_{i}\right)<F\left(X_{l}\right)$ \\
\hline 9 & Choose $F\left(X_{l}\right)$ as the best fitness \\
\hline 10 & Increment $l$ by 1 \\
\hline 11 & Repeat the steps 5 to 10 until $l<$ Maximum count \\
\hline 12 & Terminate \\
\hline
\end{tabular}

Frame 1. Pseudo code of CS algorithm

\section{CONCLUSION}

A multicast routing protocol designed with multiple QoS constraints. Multi-objective consideration is one of the challenges that most of the algorithms of multicast routing fail to follow. The proposed algorithm overcomes the above issues providing appropriate transmission along the selected suitable routes from the source to a set of destination. 


\section{CUCKOO SEARCH AND M-TREE BASED MULTICAST AD HOC ON-DEMAND DISTANCE VECTOR PROTOCOL FOR MANET}

Cuckoo Search based routing provides optimal multicasting with multiple objectives, such as energy, link lifetime, distance and delay

\section{FUTURE RESEARCH WORK}

The MANET simulation is going to be conducted in MATLAB to confirm the performance of the proposed protocol with the following parameters: energy, throughput, control overhead and PDR. Further a comparative analysis will be made with MAODV, QMRPRNS, and PDTMRP.

\section{REFERENCES}

1. R. Velmani and B. Kaarthick, "An Efficient Cluster-Tree Based Data Collection Scheme for Large Mobile Wireless Sensor Networks", IEEE Sensors Journal, vol. 15, no. 4, pp. 2377 - 2390, 2015.

2. Yun-Sheng Yen, Yi-Kung Chan, Han-Chieh Cha and Jong Hyuk Park, "A genetic algorithm for energy-efficient based multicast routing on MANETs", Computer Communications, vol. 31, no. 4, pp. 2632-2641, 2008.

3. Chia-Cheng Hu, "Bandwidth-satisfied multicast trees in largescale ad-hoc networks", Wireless Networks, vol. 16, no. 3, pp. 829-849, 2010.

4. Shiow-Fen Hwang, Yi-Yu Su, Kun-Hsien Lu and Chyi-Ren Dow, "A Cluster-Based Approach for Efficient Multi-Source Multicasting in MANETs", Wireless Personal Communication, vol. 57, no. 2, pp. 255-275, 2011.

5. Moonseong Kim, Hyunseung Choo and Matt W.Mutka, "On QoS multicast routing algorithms using k-minimum Steiner trees", Information Sciences, vol. 238, pp. 190-204, 2013.

6. $\mathrm{Xu} \mathrm{Li}$, Tianjiaot $\mathrm{Li}$, Yingt $\mathrm{Li}$ and Van Tang, "Optimized Multicast Routing Algorithm Based on Tree Structure in MANETs", China Communications, vol. 11, no. 2, pp. 90-99, 2014.

7. Vincenzo Maniscalco, Silvana Greco Polito and Antonio Intagliata, "Binary and $\mathrm{m}$-ary encoding in applications of treebased genetic algorithms for QoS routing", Soft Computing, vol. 18, no. 9, pp. 1705-1714, 2014.

8. N. C. Wang, "Power-aware dual-tree-based multicast routing protocol for mobile ad hoc networks", IET Communication, vol. 6, no. 7, pp. 724-732, 2012.

9. Ajay Kumar Yadav and Sachin Tripathi, "QMRPRNS: Design of QoS multicast routing protocol using reliable node selection scheme for MANETs", Peer-to-Peer Networking and Application, pp. 1-13, 2016.

10. Kong Sun and Chen Zeng qiang, "Tree-based differential evolution algorithm for QoS multicast routing", The Journal of China Universities of Posts and Telecommunications, vol. 18, no. 4, pp. 76-81, 2011.

11. Hu Wang, Xiangxu Meng, Shuai Li and Hong Xu, "A treebased particle swarm optimization for multicast routing", Computer Networks, vol. 54, no. 15, pp. 775-786, 2010.

12. I-Ta Lee, Guann-Long Chiou and Shun-Ren Yang, "A cooperative multicast routing protocol for mobile ad hoc networks", Computer Networks, vol. 55, no. 10, pp. 407-424, 2011.

13. Rajashekhar Biradar, Sunilkumar Manvi and Mylara Reddy, "Link stability based multicast routing scheme in MANET", Computer Networks, vol. 54, no. 7, pp. 1183-1196, 2010.

14. Rajashekhar C. Biradar and Sunilkumar S. Manvi, "Review of multicast routing mechanisms in mobile ad hoc networks", Journal of Network and Computer Applications, vol. 35, no. 1, pp. 221-239, 2012.

15. Ahmed Younes, "Multicast routing with bandwidth and delay constraints based on genetic algorithms", Egyptian Informatics Journal, vol. 12, no. 2, pp. 107-114, 2011.
16. Kumar Viswanath, Katia Obraczka and Gene Tsudik, "Exploring Mesh and Tree-Based Multicast Routing Protocols for MANETs", IEEE Transactions on Mobile Computing, vol. 5, no. 1 , pp. $28-42,2006$

17. Sung Ju Lee, William Su and Mario Gerla, "On-Demand Multicast Routing Protocol in Multihop Wireless Mobile Networks", Mobile Networks and Applications, vol. 7, no. 6, pp. 441-453, 2002.

18. Mohammad M. Qabajeh, Aisha H. Abdalla, Othman Khalifa and Liana K. Qabajeh, "A Tree-based QoS Multicast Routing Protocol for MANETs", In proceedings of IEEE 4th International Conference on Mechatronics (ICOM), pp. 17-19, 2011.

19. Chang Yeong Oh, Jongho Park and Jihyoung Ahn, "Treebased Multicast Protocol using Multi-point Relays for Mobile Ad Hoc Networks", In proceedings of IEEE 2011 Third International Conference on Ubiquitous and Future Networks (ICUFN), pp. 174-178, 2011.

20. Hasan Abdulwahid, Bin Dai, Benxiong Huang and Zijing Chen, "Scheduled-Links Multicast Routing Protocol in MANETs", Journal of Network and Computer Applications, vol. 63, pp. 56-67, 2015.

21. Mohammad Reza Effat Parvar, Mehdi EffatParvar, and Mahmoud Fathy, "Improvement of on Demand Multicast Routing Protocol in Ad Hoc Networks to Achieve Good Scalability and Reliability", Lecture Notes in Computer Science, vol. 5073, pp. 446-457, 2008.

22. Xin-She Yang, Suash Deb, "Engineering Optimisation by Cuckoo Search", Int. J. Mathematical Modelling and Numerical Optimisation, Vol. 1, No.4, pp. 330-343, 2010.

23. Satish Chander, P. Vijaya, Praveen Dhyani, "Fractional Lion Algorithm - An Optimization Algorithm for Data Clustering", Journal of Computer Science, Vol. 12, no. 7, pp. 323-340, 2016.

24. Mamatha Balachandra, K. V. Prema, Krishnamoorthy Makkithay, " Multiconstrained and multipath QoS aware routing protocol for MANETs", Wireless Networks, Vol. 20, no. 8, pp. 2395-2408, November 2014

25. P Yadhav, "Dimensionality Reduction of Weighted Word Affinity Graph using Firefly Optimization", International Journal of Engineering Research \& Technology, Vol. 3, no. 10, pp. 1324-1327, 2014.

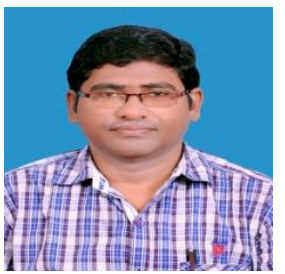

Madhu Babu D. Received his M.Sc. Degree from S.V.U, Tirupati in, 2000 and M.E degree from Satyabama University, Chennai in 2010 Currently he is pursuing Ph.D. under supervision of Dr. M. Ussenaiah, Asst.Professor, V.S.University, Nellore, AP, Indian. His research area is wireless mobile ad-hoc network.

Dr. M. Usseanaiah received his MCA degree from SKD University,

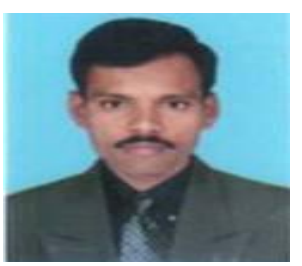
Ananthapuram and Ph.D. degree from SKD university, Ananthapuram, A.P, India Currently, he is an Assistant Professor in Computer Science Department at V.S.University, Nellore, AP, India. His research interests are in Computer Networks, wireless sensor networks, wireless Ad-hoc networks. 